\title{
Chronic Disseminated Histoplasmosis in an Immunocompetent Man Presented as Bilateral Adrenal Masses with Partial Adrenocortical Insufficiency - A Rare Condition

\author{
ABM SARWAR-E-ALAM ${ }^{\mathrm{a}}, \mathrm{Z} \mathrm{HASAN}^{\mathrm{b}}$, MAI KHAN ${ }^{\mathrm{c}}$, MA ZULKIFL $^{\mathrm{d}}$, ATM SAMDANI $^{\mathrm{e}}$,
} MT ISLAM ${ }^{\text {f }}$, SH KHAN ${ }^{\mathrm{g}}$, KB ROY ${ }^{\mathrm{h}}$
}

\begin{abstract}
Summary:
Bilateral adrenal masses caused by histoplasmosis is a rare condition. To our knowledge this is the first case report of bilateral adrenal enlargement by histoplasmosis in Bangladesh.

We report a case of 75 years old man who presented with low grade fever, night sweating, anorexia and progressive weight loss for 9 months prior to admission in Square Hospital. Clinical profile was unremarkable apart from low grade fever (with maximum temperature recorded up to
\end{abstract}

a. Dr. A B M Sarwar-e-Alam, FCPS (Internal Medicine), Consultant Physician, Department of Internal Medicine, Square Hospitals Limited, Dhaka

b. Prof. Zahidul Hasan, M Phil, MPH, Consultant Microbiologist, Pathology \& Laboratory Medicine, Square Hospitals Limited, Dhaka

c. Prof. Md. Aminul Islam Khan, Board certified in Pathology, (Iran), Consultant Pathologist, Pathology \& Laboratory Medicine, Square Hospitals Limited, Dhaka

d. Dr. MAZulkifl, FCPS (Surgery), FRCS ( England), Consultant Urologist, Department of Urology, Square Hospitals Limited, Dhaka

e. Dr. ATM Samdani, MD (DU), Associate Consultant, Radiology \& Imaging, Square Hospitals Limited, Dhaka

f. Dr. Mohd Tariqul Islam, M Phil. Associate Consultant Pathologist, Pathology \& Laboratory Medicine, Square Hospitals Limited, Dhaka

g. Sayeed Husain Khan, M Phil. Specialist, Pathology \& Laboratory Medicine, Square Hospitals Limited, Dhaka

h. Dr. Kalyan Broto Roy, MBBS. RMO- Internal Medicine, Square Hospitals Limited, Dhaka

Address of correspondence to: Dr. A B M Sarwar-e-Alam, FCPS (Internal Medicine), Consultant Physician, Department of Internal Medicine, Square Hospitals Limited, Dhaka, Mobile: 01712941697 E-mail:<drsarwaralam@hotmail.com $><$ drsarwaralam@squarehospital.com $>$ Received: 6 October 2010 Accepted: 20 September 2011 $101^{\circ} \mathrm{F}$ ) and low blood pressure with significant postural drop. Plasma cortisol (morning and evening) and glucose were normal but short ACTH stimulation test revealed low plasma cortisol response. Abdominal ultrasound gave a definite clue of left adrenal mass which was later found as bilateral adrenal masses of significant size by abdominal CT scan.

Initially Gomori's Methenamine Silver (GMS) stain of CT guided FNAC of the left adrenal mass showed small budding yeast morphologically consistent with histoplasmosis. A second CT guided FNAC of both adrenal masses also showed similar findings which was finally confirmed by fungus culture as Histoplasma capsulatum. The patient was then treated with injection amphotericin-B followed by oral itraconazole and fludrocortisone and he responded well.

Key words: Immunocompetent elderly man, bilateral adrenal masses, CT-guided FNAC, Histoplasma capsulatum, Histoplasmosis, Partial adrenal insufficiency.

(J Bangladesh Coll Phys Surg 2011; 29: 235-240)

Introduction:

Histoplasmosis is a fungal disease that is endemic in USA, Central Africa and South of Sahara and sporadic in Africa and Far East ${ }^{1-4}$. Human cases are rare outside endemic areas. The true prevalence of histoplasmosis in South East Asia is not known and pockets of endemicity probably exist throughout the region.

The disease is caused by Histoplasma capsulatum which has two forms: spore (saprophytic) and yeast (infective) form. In humans, spores usually enter into the body through inhalation via lungs where it germinates into yeast form and rarely through inoculation (skin) and sometimes by some unknown routes. The spores are found in contaminated soil and their growth is facilitated by excreta of birds and bats e.g. bat guano ${ }^{3,4}$. 
In most (99\%) cases the infected persons show no overt manifestation. However immunosuppressed persons and persons with extremes of ages may develop acute or chronic pulmonary infection or disseminated form of the disease ${ }^{3,4,5}$. Chronic disseminated histoplasmosis rarely occurs in normal (immunocompetent) adults. Recurrent illness interspersed between asymptomatic periods ranging from several years up to two decades may be seen in some patients cell immunity plays the predominant role in recovery from histoplasmosis 6,7 . Diagnosis of disseminated histoplasmosis depends on a high index of suspicion, recognition of the common mode of presentation, histopathological examination and microbiological confirmation by obtaining samples such as sputum, bronchoalveolar lavage, blood, bone marrow or any other suspected tissue.

\section{Case Report:}

A 75 year old Bangladeshi male, farmer of middle class family hailed from Gopalgonj district previously in good health was admitted in Square Hospitals Limited, Dhaka, on $5^{\text {th }}$ October 2009 with 9 months history of anorexia, progressive weight loss and irregular low grade evening fever with chills which subsided with mild sweating. He had no previous history of tuberculosis, histoplasmosis or travel abroad. Prior to admission here he was admitted in several hospitals of Dhaka city without any improvement. He received antibiotic therapy without success.

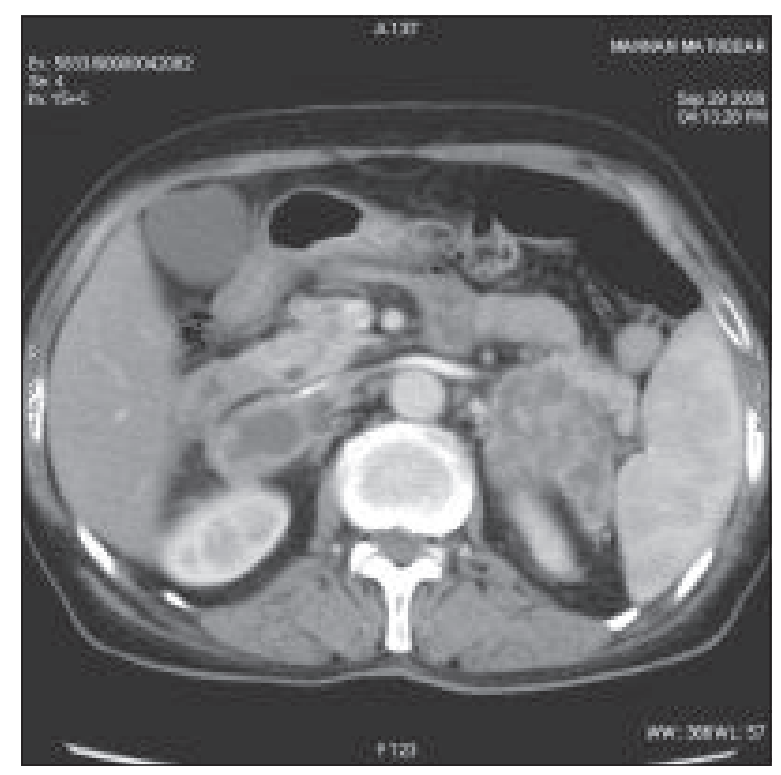

Fig.-1 (Axial View): Contrast CT Abdomen showingbilateral adrenal masses.
On examination he was ill-looking, mildly pale and emaciated with weight of $50 \mathrm{~kg}$. There was no peripheral lymphadenopathy. He was having postural hypotension (basal lying pressure was 100/62 $\mathrm{mmHg}$ while standing pressure was $70 / 55 \mathrm{mmHg}$ ) with pulse $100 / \mathrm{min}$. Fever pattern was irregular with maximum temperature recorded up to $101^{\circ} \mathrm{F}$ in the evening. Cardiac and pulmonary examinations were normal. Abdominal and neurological examinations also revealed no abnormality.

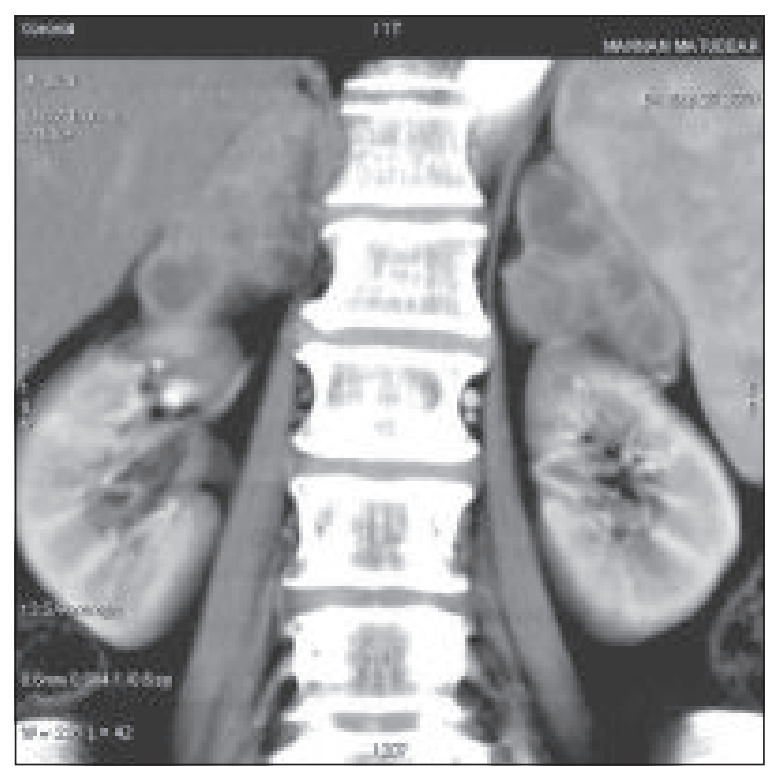

Fig.-2 (Coronal View): Contrast CT Abdomen showing - bilateral adrenal masses.

Laboratory investigations showed normal serum cortisol levels in the morning $(14 \mu \mathrm{g} / \mathrm{dl})$ and evening $(11.9 \mu \mathrm{g} /$ dl) with normal serum potassium $4.1 \mathrm{mmol} / \mathrm{L}$ and serum CRP $24.2 \mathrm{mg} / \mathrm{L}$. Serology for HIV was negative. There was no lymphopenia. Mantoux test was negative (02 $\mathrm{mm}$ size) and the chest X-ray was normal. However, there was mild elevation of ESR ( $41 \mathrm{~mm}$ in first hour). Abdominal ultrasonography revealed a left adrenal mass (Size $7.21 \mathrm{X} 4.18 \mathrm{~cm}$ ). CT scan of both kidneys and supra-renal glands with I/V contrast showed bilateral, almost symmetrical heterogeneous well defined adrenal masses [ Left adrenal mass approx. $68 \mathrm{~mm}$ (CC) X 61 mm (AP) X 48 mm (TR), right adrenal mass approx. 64 mm (CC) X $61 \mathrm{~mm}$ ( AP) X 34 mm (TR) ] consistent with likelihood of tuberculosis or metastasis as shown in figure $2 \& 3$. CT guided FNAC of the left adrenal 
mass was done and sent for histopathological examination. GMS stain showed yeast like bodies extracellularly as well as within macrophages along with necrotic materials containing numerous neutrophils and macrophages as shown in figure 6. Morphologically these features were consistent with adrenal abscess with histoplasmosis. Repeat CT guided aspiration biopsy of both adrenal masses from posterior approach using a LP needle ( size 23) was performed. GMS stain of the both adrenal aspirates again showed the same findings and fungus culture of the specimen confirmed diagnosis of Histoplasma capsulatum as shown in figure 7. Short ACTH stimulation test was also done. On the day of test, morning plasma cortisol was $9.9 \mu \mathrm{g} / \mathrm{dl}$ ( normal 4.2 to $38.4 \mu \mathrm{g} / \mathrm{dl}$ ) and morning plasma ACTH level was $34.60 \mathrm{pg} / \mathrm{ml}$ (normal 8.3 to $57.8 \mathrm{pg} / \mathrm{ml}$ ). After Injection ACTH $250 \mu \mathrm{gm} \mathrm{I} / \mathrm{M}$, plasma cortisol increased to 12.2 $\mu \mathrm{g} / \mathrm{dl}$ at the end of 45 minutes.

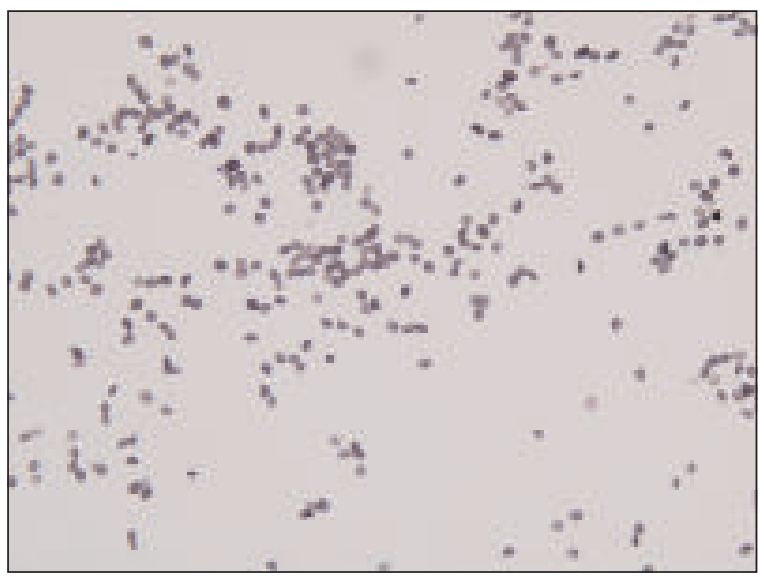

Fig.-3: Histoplasma-Gomori's Methenamine Silver (GMS) Stain $x 100$

The patient was treated initially with 5 days course of amphotericin-B I/V infusion daily followed by oral itraconazole $200 \mathrm{mg}$ three times daily after food for three days and thereafter $100 \mathrm{mg}$ twice daily to be continued for one year. In addition, he was given oral fludrocortisone $0.1 \mathrm{mg}$ once daily for persistent low BP with significant postural drop. He was discharged from hospital on $19^{\text {th }}$ October 2009 while he was improving. He came for follow up in medical OPD on $10^{\text {th }}, 24^{\text {th }}$ and $27^{\text {th }}$ week of treatment. During follow up at $24^{\text {th }}$ week, he was found free from all of the previous symptoms but with a few new problems. These were systolic hypertension without postural drop ( BP 160/

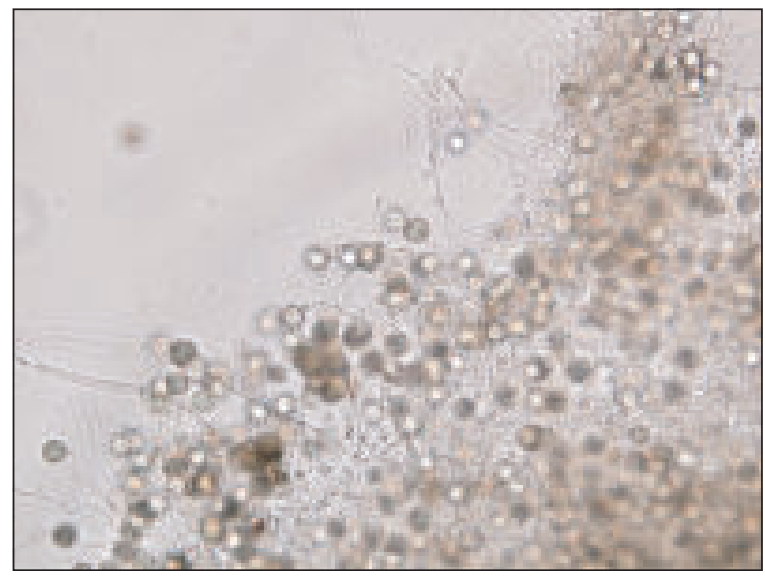

Fig.-4: Growth of Histoplasma capsulatum in Sabouraud's Agar media

$80 \mathrm{~mm}$ of $\mathrm{Hg}$ and pulse 48 / minute), edema in lower limbs and hypokalemia ( $2.8 \mathrm{mmol} / \mathrm{L})$. All relevant laboratory investigations were normal except serum potassium $(2.8 \mathrm{mmol} / \mathrm{L}), \mathrm{CRP}(13.1 \mathrm{mg} / \mathrm{L}), \mathrm{ESR}(44 \mathrm{~mm}$ in first hour) and $\mathrm{Hb}(11 \mathrm{gm} \%)$. During this follow up, dose of Fludrocortisone was reduced by $0.05 \mathrm{mg}$ every alternate day but other treatment was kept same .During his last follow up ( $27^{\text {th }}$ Week) he was totally asymptomatic with normalization of blood pressure ( $120 / 80 \mathrm{mmHg}$ ), serum potassium $(4.5 \mathrm{mmol} / \mathrm{L})$ and disappearance of peripheral oedema.

\section{Discussion :}

Disseminated histoplasmosis is a rare and potentially fatal disease caused by dimorphic soil fungus Histoplasma capsulatum ${ }^{8}$. This fungus has a particular predilection for adrenal cortex causing adrenal enlargement .It is more common in male with male to female ratio of 3-10:1. Due to its non-specific presentation, disseminated histoplasmosis usually creates diagnostic dilemma to the clinicians. The common clinical features include fever, weight loss, anorexia and malaise. In endemic countries useful clues in the diagnosis of disseminated histoplasmosis are one or more of following features : unilateral or bilateral adrenal enlargement in USG / CT scan, postural hypotension, electrolyte abnormalities, hypoglycaemia .But in country like ours, we should think of more common diseases such as tuberculosis, secondary metastasis or sarcoidosis before considering disseminated histoplasmosis ${ }^{9}$. This patient presented with long duration of fever, night sweat, weight loss, 
anorexia, along with low blood pressure (100/62 mm of $\mathrm{Hg}$ ) with significant postural drop, which suggests involvement of both adrenal glands. However, we got definite evidence of adrenal involvement by abdominal USG and CT scan suggesting either of metastatic malignancy, chronic disseminated tuberculosis or deep mycosis at this age ${ }^{10}$.

On ultrasound, adrenal masses are usually hypoechoic ( as seen in this case) and can be homogenous or heterogenous in texture with characteristic feature of maintenance of triangular shape ${ }^{11,12}$. The most definite way to get the final diagnosis is to perform CT or Ultrasound guided FNAC or open biopsy of the adrenal mass ${ }^{13,14}$ and send for histopathological and microbiological examinations. We did FNAC for our diagnosis and found histoplasmosis in both the adrenal masses. So this is a case of chronic disseminated histoplasmosis with bilateral adrenal masses ${ }^{13-16}$. It has been observed in several studies that adrenal involvement in the disseminated form is relatively common in HIV-negative patients than in HIV-positive ones. Moreover Kumar et al described 9 cases of adrenal histoplasmosis in India; all of them were HIVnegative ${ }^{17-19}$.

As the basal morning and evening plasma cortisol were normal, we performed short ACTH stimulation test to see stimulated plasma cortisol response .It revealed partial glucocorticoid deficiency because ACTH stimulation did not increase plasma cortisol $>18 \mu \mathrm{g} / \mathrm{dl}$ (i.e., low ACTH stimulated cortisol response) ${ }^{13,20}$. In normal adrenal functional state, plasma cortisol is increased to $>18 \mu \mathrm{g} / \mathrm{dl}$ at or after 45 minutes following intramuscular injection ACTH. In clinically overt case, morning plasma cortisol should be below $3 \mu \mathrm{g} / \mathrm{dl}$. On the contrary if morning plasma cortisol is $18 \mu \mathrm{g} / \mathrm{dl}$ or more and then it virtually excludes Addison's disease .Normal plasma ACTH level indicates normal anterior pituitary .The other main component of adrenal cortex is mineralocorticoid which was insufficient in our patient as evident by significant postural drop of blood pressure . This was corrected with fludrocortisone therapy. We can conclude that so far adrenal function is concerned, this patient had partial glucocorticoid and full mineralocorticoid deficiency. Involvement of adrenal gland is common $(80-90 \%)$ in chronic disseminated histoplasmosis. However, only about half ( $40 \%$ ) develop adrenal insufficiency. Among them only $10 \%$ of cases develop full blown adrenal insufficiency ${ }^{8}$.

Disseminated histoplasmosis may affect almost any system in immunocompromised individuals or at age extremities. Histoplasmosis presenting as bilateral adrenal enlargement has been previously described $9,15,16,21,23$. However, to our knowledge, this case of bilateral adrenal histoplasmosis with partial adrenal insufficiency in an immunocompetent host is rare worldwide $11,16,21,23$.

In Bangladesh, so far no case was reported as a patient with bilateral adrenal masses due to histoplasmosis. This man did not travel to any other country and hence it is difficult to presume how did he acquire this disease. Further search in his local area, Gopalgonj may reveal other cases of histoplasmosis. Our patient did not develop disseminated histoplasmosis by re-infection because there is no previous history of documented histoplasmosis. Logically this is a case of re-activation of latent histoplasmosis, where organisms were in quiescent phase following recovery from initial episode of asymptomatic manifestation.. The reactivation is commonly associated with immunosuppressive diseases such as diabetes, but in some cases age is the only risk factor probably due to a selective defect in the cell mediated immunity against the Histoplasma ${ }^{17}$. This patient was neither diabetic nor immunosupressed.

Our patient responded to treatment by amphotericin-B and was followed by oral itraconazole and fludrocortisone. During follow up we found that this patient had developed signs of excess fludrocortisone therapy (hypokalemia, hypertension and peripheral oedema). These side effects disappeared completely with reduction of fludrocortisone dose .

Treatment is indicated for all patients with disseminated histoplasmosis. Clinical practice guidelines for the management of patients with histoplasmosis were updated in 2007 by the Infectious Disease Society of America $^{23}$. In the majority of cases, the choice of acceptable therapy is one of lipid formulations of Amphotericin-B (liposomal amphotericin-B, deoxycholate amphotericin-B or lipid complex amphotericin-B ) I/V daily till patient becomes afebrile or at least shows improvement of fever ( this usually takes 3 to 7 days) followed by oral itraconazole $200 \mathrm{mg}$ three times daily with food for three days ( to achieve steady serum concentration more rapidly) ${ }^{21}$. Thereafter 
the maintenance dose of $200 \mathrm{mg}$ once or twice daily. We could not detect serum concentration of Itraconazole due to lack of facilities. Liposomal amphotericin -B is better than other formulations. The optimal duration of Itraconazole therapy is not known. Therapy for one to two years is preferred because it may reduce the risk of relapse $9,15,20$. Chronic maintenance therapy may be needed in immunosuppressed individuals and those who relapse after one year of treatment. Fluconazole is not as active against Histoplasma capsulatum as itraconazole and is associated with increased side effects and development of resistance ${ }^{9}$. That is why fluconazole was not given to our patient.

Concentration of Histoplasma antigen in urine and serum fall with effective therapy $7,24,25$. Failure to do so indicates ineffective therapy ${ }^{5}$. In responders, serum and urine histoplasma antigen should be performed at 4-6 months intervals. The antigen detection tests are not available in our country. Most ( $~ 90 \%$ ) cases of adrenal insufficiency are partial and do not require cortisol replacement therapy. Reversal of adrenal dysfunction to normal has been described after prolonged antifungal treatment 20,22 .

Disseminated histoplasmosis if remains undiagnosed or left untreated is usually fatal. Early diagnosis and treatment leads to favorable outcome 26 .

\section{Conclusion:}

Any case of prolonged fever without localizing symptoms and signs, and /or adrenal insufficiency should arouse the suspicion of chronic histoplasmosis infection in addition to consideration of other causes of chronic infections and malignancies. The clue to diagnosis may be facilitated by the detection of bilateral or unilateral adrenal enlargement on CT scan. Confirmation depends upon culture and biopsy of the infected tissue samples .CT guided FNAC of the adrenal gland is a safe, accurate, inexpensive and effective method in the diagnosis of adrenal histoplasmosis. Continuation of anti-fungal therapy for at least one year along with monitoring of adrenal insufficiency is the recommended method of management .

\section{References:}

1 Chu, JH, Feudtner, C, Heydon, K, et al. Hospitalizations for endemic mycoses: a population-based national study. Clin Infect Dis. 2006; 42:822.
2 Goodwin, RA Jr, Shapiro, JL, Thurman, GH, et al. Disseminated histoplasmosis: Clinical and pathologic correlations. Medicine (Baltimore). 1980; 59:1.

3 Adderson E. Histoplasmosis. The Pediatric Infectious Disease Journal. 2006; 25: 73-74.

4 Wheat LJ. Histoplasmosis: a review for clinicians from nonendemic areas. Mycoses. 2006; 49:274-282.

5 Wheat, LJ. Improvements in diagnosis of histoplasmosis. Expert Opin Biol Ther. 2006; 6:1207.

6 Deepe, GS Jr, Bullock, WE. Histoplasmosis: A granulomatous inflammatory response. In: Inflammation: Basic Principals and Clinical Correlates, Gallin, JI, Goldstein, IM, Snyderman, R (Eds), Raven, New York. 1988; p.733

7 Wheat, LJ, Connolly-Stringfield, P, Blair, R, et al. Effect of successful treatment with amphotericin B on Histoplasma capsulatum variety capsulatum polysaccharide antigen levels in patients with AIDS and histoplasmosis. Am J Med. 1992; 92:153.

8 Schonfeld AD, Jackson JA, Smith DJ, Hurley DL. Disseminated histoplasmosis with bilateral adrenal enlargement: diagnosis by computed tomography - direct needle biopsy. Tex Medicine. April 1991; 87(4); 88 - 90 .

9 Grover SB, Midha N, Gupta M, Sharma U, Talib VH. Imaging spectrum in disseminated histoplasmosis: case report and brief review. Australasian Radiology. 2005; 49: 175-178.

10 Luning M, Hoppe E, Schopke W. Results of diagnosis of adrenal masses using percutaneous CT guided fine needle biopsy. Rofo. 1986; 144 (2); 154 - 159.

11 Kumar N, Singh S, Govil S. Adrenal histoplasmosis: clinical presentation and imaging features in nine cases. Abdominal Imaging. 2003; 28: 703-708.

12 Wilson DA, Mynyen DL, Tylk TL, Swancy CM, Muchmore HG. Sonography of adrenal glands in chronic disseminated histoplasmosis. J Ultrasound Med. 1986; 5 (2), 69 - 73.

13 Cooper MS, Stewart PM. Corticosteroid insufficiency in acutely ill patients. N Eng J Med. 2003; 348: 727-734.

14 Anderson CJ, Pitts WC, Weises LM. Disseminated histoplasmosis diagnosed by fine needle aspiration biopsy of the adrenal gland - A case report. Acta Cytol. 1989; 33 (3), $337-340$.

15 Giacaglia LR, Lin CJ, Lucon AM, Goldman J. Disseminated histoplasmosis presenting as bilateral adrenal masses. Revista do Hospital das Clínicas.1998; 53: 254-256.

16 Mahajan R, Sharma U, Trivedi N, Prasad M, Kansra U, Bhandari S, Talib VH. Histoplasma capsulatum in adrenal gland aspirate - a case report. Indian Journal Pathology \& Microbiology. 2000; 43: 165-168.

17 Kauffman C.A. Fungal infections in older adults. Clin Infect Dis. 2001;33:550-5. 
18 Wheat J. Histoplasmosis: experience during outbreaks Indianapolis and review of the literature. Medicine. 1997;76(5):339-54.

19 Wheat, J, Hafner, R, Korzun, AH, et al. Itraconazole treatment of disseminated histoplasmosis in patients with the acquired immunodeficiency syndrome. Am J Med. 1995; 98:336.

20 Washburn RG, Bennett JE. Reversal of Adrenal Glucocorticoid Dysfunction in a Patient with Disseminated Histoplasmosis. Ann Intern Med. 1989;110: 86-87.

21 Dismukes, WE, Bradsher, RW Jr, Cloud, GC, et al. Itraconazole therapy for blastomycosis and histoplasmosis. Am J Med. 1992; 93:489.

22 Lee J, Jones PH, Trowell JE, Whitear WP, Williams PF. Hypoadrenal crisis caused by disseminated histoplasmosis. The Journal of Infection. 1993; 27: 181-183.
Wheat, LJ, Freifeld, AG, Kleiman, MB, et al. Clinical practice guidelines for the management of patients with histoplasmosis: 2007 update by the Infectious Diseases Society of America. Clin Infect Dis. 2007; 45:807.

24 Hecht, FM, Wheat, J, Korzun, AH, et al. Itraconazole maintenance treatment for histoplasmosis in AIDS: A prospective, multicenter trial. J Acquir Immune Defic Syndr Hum Retrovirol. 1997; 16:100.

25 Wheat, J, Hafner, R, Wulfson, M, et al. Prevention of relapse of histoplasmosis with itraconazole in patients with the acquired immunodeficiency syndrome. Ann Intern Med. 1993; 118:610.

26 Subramanian S, Abraham OC, Rupali P, Zachariah A, Mathews MS, Mathai D Disseminated histoplasmosis. J Assoc Physicians India. 2005; 53:185-189. 